\title{
What makes the chemical diversity of ocean worlds in the Solar System?
}

\author{
YASUHITO SEKINE ${ }^{1}$, SHUYA TAN ${ }^{2}$, YUHIKO \\ AOYAMA $^{3}$, TAKAYUKI TANIGAWA ${ }^{4}$ AND MASAHIRO \\ IKOMA $^{5}$ \\ ${ }^{1}$ Tokyo Institute of Technology, Earth-Life Science Institute \\ ${ }^{2}$ Tokyo Institute of Technology \\ ${ }^{3}$ Tsinghua University \\ ${ }^{4}$ National Institute of Technology, Ichinoseki College \\ ${ }^{5}$ University of Tokyo \\ Presenting Author: sekine@elsi.jp
}

Planetary habitability can be defined as a global-scale system that develops ingredients of life-liquid water, organic matter, and energy - and provides them to life continuously through hydrogeochemical cycles over geological time. Owing to the previous Solar System explorations, we have found lines of geophysical, geochemical, and geological evidence that show long-term existence of liquid water on multiple Solar System bodies, including ocean worlds. However, when it comes to more know, we start to realize that there is a wide diversity in water chemistry ocean worlds. Enceladus and Ceres would have alkaline, ammonia-rich subsurface oceans; whereas, Europa's ocean might not be enriched in these volatiles. Ganymede and Titan are similar in size and mass; however, only Titan has a thick methane-rich atmosphere.

Here, we discuss what are key geophysical and geochemical processes for making the diversity of chemistry in the ocean worlds in the Solar System. Availability of both chemical energy and organic matter on these Solar System bodies should be largely affected by the diversity of chemistry. Thus, the diversity of chemistry is likely to determine the diversity of potential biospheres there. In this presentation, we discuss the causative mechanisms of the chemical dichotomy of ocean worlds between the Jovian and Saturnian systems. We discuss the availability of volatiles on the Jovian system vs that of the Saturnian system, by focusing on the formation of these satellite systems around Jupiter and Saturn in the early Solar System. Then, we discuss the evolution of oceanic chemistry, by focusing on effects of seafloor rock compositions of ocean worlds to determine the chemical diversity. 